\title{
Serious Adverse Event
}

National Cancer Institute

\section{Source}

National Cancer Institute. Serious Adverse Event. NCI Thesaurus. Code C41335.

Any expected or unexpected adverse event, related or unrelated to the intervention, occurring at any agent dose, any phase of product or procedure testing, that results in any of the following outcomes: death, a life-threatening adverse event, requires inpatient hospitalization (not required as part of the treatment) or prolong ation of existing hospitalization, a persistent or significant disability or incapacity, or cancer, or a congenital anomaly or birth defect. Important medical events that may not result in the listed outcomes may be considered as serious when, based upon appropriate medical judgment, they represent significant hazards or potentially serious harm to the research subject or others and may require medical intervention to prevent one of the outcomes listed in this definition. 